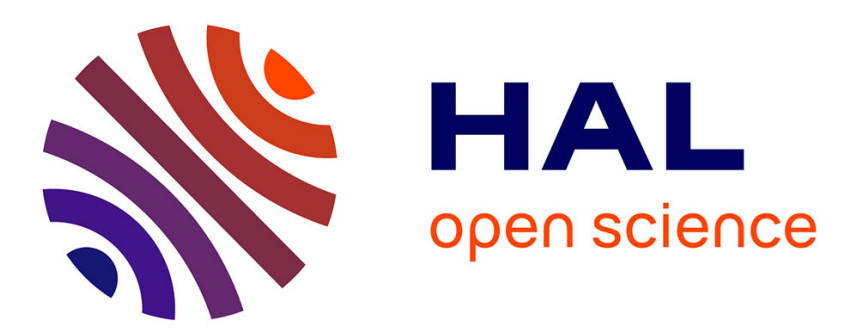

\title{
Experimental validation of an analytical model for predicting the thermal and hydrodynamic capabilities of flat micro heat pipes
}

Rémi Revellin, Romuald Rullière, Frédéric Lefèvre, Jocelyn Bonjour

\section{- To cite this version:}

Rémi Revellin, Romuald Rullière, Frédéric Lefèvre, Jocelyn Bonjour. Experimental validation of an analytical model for predicting the thermal and hydrodynamic capabilities of flat micro heat pipes. Applied Thermal Engineering, 2009, 29 (5-6), pp.1114-1122. 10.1016/j.applthermaleng.2008.06.002 . hal-00626333

\section{HAL Id: hal-00626333 \\ https://hal.science/hal-00626333}

Submitted on 26 Sep 2011

HAL is a multi-disciplinary open access archive for the deposit and dissemination of scientific research documents, whether they are published or not. The documents may come from teaching and research institutions in France or abroad, or from public or private research centers.
L'archive ouverte pluridisciplinaire $\mathbf{H A L}$, est destinée au dépôt et à la diffusion de documents scientifiques de niveau recherche, publiés ou non, émanant des établissements d'enseignement et de recherche français ou étrangers, des laboratoires publics ou privés. 


\section{Accepted Manuscript}

Experimental validation of an analytical model for predicting the thermal and hydrodynamic capabilities of flat micro heat pipes

Rémi Revellin, Romuald Rullière, Frédéric Lefèvre, Jocelyn Bonjour

PII:

S1359-4311(08)00260-3

DOI:

10.1016/j.applthermaleng.2008.06.002

Reference:

ATE 2530

To appear in:

Applied Thermal Engineering

\section{APPLIED}

THERMAL

ENGINEERING

6 December 2007

Received Date:

28 May 2008

Revised Date:

2 June 2008

Accepted Date:

Please cite this article as: R. Revellin, R. Rullière, F. Lefèvre, J. Bonjour, Experimental validation of an analytical model for predicting the thermal and hydrodynamic capabilities of flat micro heat pipes, Applied Thermal Engineering (2008), doi: 10.1016/j.applthermaleng.2008.06.002

This is a PDF file of an unedited manuscript that has been accepted for publication. As a service to our customers we are providing this early version of the manuscript. The manuscript will undergo copyediting, typesetting, and review of the resulting proof before it is published in its final form. Please note that during the production process errors may be discovered which could affect the content, and all legal disclaimers that apply to the journal pertain. 


\title{
Experimental validation of an analytical model for predicting the thermal and hydrodynamic capabilities of flat micro heat pipes.
}

Rémi Revellin, Romuald Rullière, Frédéric Lefèvre * Jocelyn Bonjour

Centre de Thermique de Lyon (CETHIL) UMR 5008 CNRS-INSA-Univ. Lyon 1 Bât. Sadi Carnot, INSA-Lyon, F-69621 Villeurbanne Cedex, France

\begin{abstract}
An analytical model by Lefèvre and Lallemand (1) that couples a 2D hydrodynamic model for both the liquid and the vapor phases inside a flat micro heat pipe (FMHP) and a 3D thermal model of heat conduction inside the FMHP wall has been modified. It consists of superposing two independent solutions in order to take into account the impact of evaporation or condensation on the equivalent thermal conductivity of the capillary structure. The temperature, pressure and velocity fields can be determined using Fourier solutions. The model has been experimentally validated based on literature data from a grooved FMHP. Two new correlations for the equivalent thermal conductivities during evaporation and condensation inside rectangular micro-grooves have been proposed based on a numerical database. The influence of the saturation temperature and geometry on the maximum heat flux transferred by the system is presented.
\end{abstract}

Key words: Two-phase heat spreader; Micro heat pipe; Meniscus curvature; Micro-grooves; Analytical model 


\section{Nomenclature}

$a, b, c$

$a_{c}$

$A_{\mathrm{m} 0}, A_{0 \mathrm{n}}, A_{\mathrm{mn}}$

$B, C$

$B_{\mathrm{m} 0}, B_{0 \mathrm{n}}, B_{\mathrm{mn}}$

$C_{\mathrm{m} 0}, C_{0 \mathrm{n}}, C_{\mathrm{mn}}$

D

f

$H$

$h$

$h_{\mathrm{lv}} \quad$ Latent heat of vaporization

K Permeability

$l$

FMHP dimensions

Accommodation coefficient

Fourier coefficients of $T^{*}$

Dimensionless lengths

Fourier coefficients of $\phi$

Fourier coefficients of $P$

Dimensionless parameter

Friction factor

Height

Heat transfer coefficient

Width m

$\mathrm{W} / \mathrm{m}^{2} \mathrm{~K}$

$\mathrm{J} / \mathrm{kg}$

$\mathrm{m}^{2}$

$\mathrm{m}$

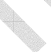


Mean absolute error $=$

$\frac{1}{N} \sum_{1}^{N}\left|\frac{\text { predicted value-numerical value }}{\text { numerical value }}\right| \times 100$

MRE

Mean relative error $=$

$\frac{1}{N} \sum_{1}^{N}\left(\frac{\text { predicted value-numerical value }}{\text { numerical value }}\right) \times 100$

P

Pressure

$\mathrm{Pa}$

$\bar{R}$

Universal gas constant

$\mathrm{kJ} / \mathrm{mol} . \mathrm{K}$

$r$

Pore radius

$\mathrm{m}$

$R$

Curvature radius

$\mathrm{m}$

$R_{0} \quad$ Curvature radius in non-working conditions

$\mathrm{m}$

$R e$

Reynolds number

$T$

Temperature

K

$T^{*}$

Dimensionless temperature

$x, y, z$

Coordinates

$\mathrm{m}$

$X, Y, Z$

Dimensionless coordinates

Greek letters

$\alpha^{*}$

Dimensionless parameter 
$\delta$

$\eta$

$\lambda$

$\mu$

$\xi$

$\rho$

$\sigma$

$\varphi_{0}$

$\phi$

Subscripts

1

2

c

cap

e

eq

f
Liquid film thickness

$\mathrm{m}$

Ratio between the evaporator and condenser areas -

Thermal conductivity

$\mathrm{W} / \mathrm{m} . \mathrm{K}$

Dynamic viscosity

Pa.s

Porosity

Density

Surface tension

Imposed heat flux

Dimensionless heat flux $\mathrm{kg} / \mathrm{m}^{3}$

$\mathrm{N} / \mathrm{m}$

$\mathrm{W} / \mathrm{m}^{2}$

Index

Index

Condenser

Capillary

Evaporator

Equivalent

Fin 
g

g

$\mathrm{h}$

int

1

$\mathrm{p}$

S

sat

V
Gravity

Groove

Hydraulic

Interfacial

Liquid

Porous medium

Solid

Saturation

Vapor 


\section{Introduction}

Flat micro heat pipes are very efficient cooling systems that are able to transfer high heat fluxes with small temperature gradients due to phase change phenomena namely, evaporation at a heat source (evaporator) and condensation at a heat sink (condenser). These devices are widely studied for the thermal management of high power electronic components (Lallemand and Lefèvre (2)), in particular because of their possible integration in silicon substrates (Kang et al. (3), Ivanova et al. (4) and Benson et al. (5)). In addition, their high level of thermal performance allowed their use in other applications such as the cooling of fuel cell systems (Rullière et al. (6)). In this last application, a micro grooved FMHP made of copper was tested and filled with methanol. Unlike conventional FMHP applications, the heat source is larger than the heat sink. The cooling system is used for its heat transfer capacity as well as its heat spreading characteristics that permits to homogenize the temperature in the core of fuel cells.

In FMHP's, the liquid returns from the condenser to the evaporator through a capillary structure made of parallel or crossed micro grooves, meshes, sintered powder wicks... Several works have been published for the thermal and hydrodynamic modeling of FMHP with longitudinal micro-grooves (Longtin et al. (7), Kim et al. (8), Sartre et al. (9) and Khrustalev and Faghri (10)). The modeling of both the liquid and the vapor flows in grooves is relatively easier than in other capillary structures because of the geometric simplicity.By the use of the Young-Laplace law in the balance equations, the location and the shape of both the liquid and the vapor in each groove section can be calculated. These last parameters are important for the modeling of the thermal performances 
of those devices because the variation of the liquid thickness that occurs along the grooves affects the liquid film resistance in the evaporator and condenser areas. Most of the theoretical works published on FMHP are not validated with experimental data or only validated with thermal measurements outside the FMHP. Actually, the measurement of hydrodynamic parameters (liquid and vapor velocities or pressures) is difficult inside micro grooves.

Rullière et al. (6) used a confocal microscope to measure the meniscus curvature radius along the grooves of a FMHP made of copper and filled with methanol. This measurement was used to validate a two-phase flow model based on the conservation equations and the Young-Laplace equation. In fact, the meniscus curvature radius is related to the vapor and liquid pressures through the Young-Laplace Law. The numerical model allows the calculation of the meniscus curvature radius, the liquid and vapor pressures and the liquid and vapor velocities along the FMHP. The comparison between the experimental results and the model shows the good ability of the numerical model to predict the meniscus curvature radii, from which the maximum heat transfer capability of the FMHP is depending.

More recently, Lefèvre et al. (11) developed a thermal model of the FMHP coupled to the above-mentioned hydrodynamic model. The meniscus curvature radius in the grooves is an input parameter of the thermal model. This model allows the calculation of the heat transported by a grooved FMHP and the temperature field in its wall. It has been validated by using temperature data obtained with several thermistors located along the FMHP wall. A good agreement has been found between the experimental and the calculated temperatures. Thus, the coupled hydrodynamic and thermal models were validated by using both thermal and hydrodynamic measurements. 
More general models have been developed for complex capillary structures such as meshes or sintered powder wicks. Huang and Lin (12) and Qin and Liu (13) introduced the Darcy's law in the balance equation to calculate, through an analytical solution, both the pressure and the velocity field in the porous medium. Lefèvre and Lallemand (1) improved the Huang and Lin (12) and Qin and Liu (13) models by taking into account both the liquid and the vapor flows inside a FMHP and the temperature field in the wall. An analytical solution for the pressures and velocities of both the liquid and the vapor is coupled to a thermal analytical solution for the temperature field in the wall. The model parameters are the permeability, the porosity and the equivalent thermal conductivity of the capillary structure that are considered to be constant over the whole FMHP.

In the present work, the analytical model developed by Lefèvre and Lallemand (1) is completed to take into account the difference between the equivalent thermal conductivities in the evaporation and condensation areas. As a matter of fact, the equivalent thermal conductivity of capillary structures like axial grooves is significantly different in the evaporator and condenser regions due to the different heat and mass transfer processes occurring. Correlations are developed to calculate the equivalent thermal conductivity of grooves in both the evaporator and condenser sections. The results are compared to the experimental data by Rullière et al. (6).

\section{Governing equations and boundary conditions}

The geometry of the flat micro heat pipe consists of a flat plate of $a \times b$ rectangular dimensions as shown in Fig. 1. The electronic components and the 
heat sinks can be located anywhere in contact with the FMHP wall and have different heat dissipations. The rest of the surface is adiabatic. The FMHP wall whose thickness is $c$, has a constant thermal conductivity $\lambda_{\mathrm{s}}$. The capillary structure is modeled by considering a porous medium of permeability $K$, thermal conductivity $\lambda_{\mathrm{p}}$ and thickness $H_{\mathrm{p}}$. The liquid inside the porous medium has a dynamic viscosity $\mu_{1}$, a thermal conductivity $\lambda_{1}$ and a density $\rho_{\mathrm{l}}$. The equivalent conductivity of the porous medium filled with liquid is equal to $\lambda_{\text {eq }}$. The thickness of the vapor space is equal to $H_{\mathrm{v}} \cdot \lambda_{\mathrm{v}}$ and $\mu_{\mathrm{v}}$ are respectively the vapor thermal conductivity and the dynamic viscosity. The thermal properties are calculated at the saturation temperature $T_{\text {sat }}$ which is assumed to be uniform inside the FMHP (equal to the vapor temperature).

\subsection{Thermal model and analytical solution}

A 3D thermal model has been developed assuming that all the surfaces of the FMHP are well insulated except the heat sources and heat sinks. The 3D heat conduction equation is analytically solved for the FMHP wall. Heat transfer by conduction and phase change in the porous medium is taken into account through a Fourier boundary condition at $z=0$. Two different values of capillary structure equivalent conductivities are considered here, namely one for the structure filled with an evaporating liquid and the other for the structure in which condensation takes place. Thus, the analytical solution for the temperature field inside the wall is obtained as the sum of two independent solutions:

$$
T=T_{\mathrm{e}}+T_{\mathrm{c}}
$$


where $T_{\mathrm{e}}$ and $T_{\mathrm{c}}$ are respectively the temperature field due to heat transfer from the heat sources to the vapor and the temperature field due to heat transfer from the vapor to the heat sink. It should be emphasized that this superposition of two independent solutions is exact only if the distance between the heat sources and the heat sinks is large enough to avoid superposition of evaporating and condensing areas.

At $z=c$, the boundary condition is assumed to be an imposed heat flux $\varphi_{0}$ at the heat sources, an imposed heat flux equal to $-\eta \times \varphi_{0}$ at the heat sink $(\eta$ being the ratio between the heat source and the heat sink areas) and a heat flux equal to zero in the adiabatic area.

Introduce the non-dimensional coordinates $X, Y$ and $Z$ :

$$
X=\frac{x}{a} ; Y=\frac{y}{b} ; Z=\frac{z}{c}
$$

and the non-dimensional lengths:

$$
B=\frac{b}{a} ; C=\frac{c}{a}
$$

The non-dimensional temperature field inside the FMHP wall, $T^{*}$, can thus be expressed as the sum of two independent and separated solutions:

$$
T^{*}=T_{\mathrm{e}}^{*}+T_{\mathrm{c}}^{*}
$$

$T_{e}^{*}=\lambda_{s}\left(T_{e}-T_{\mathrm{sat}}\right) / \varphi_{0} \mathrm{c}$ is the non-dimensional temperature solution of the following 3D steady-state heat conduction equation: 


$$
\frac{\partial^{2} T_{\mathrm{e}}^{*}}{\partial X^{2}}+\frac{1}{B^{2}} \frac{\partial^{2} T_{\mathrm{e}}^{*}}{\partial Y^{2}}+\frac{1}{C^{2}} \frac{\partial^{2} T_{\mathrm{e}}^{*}}{\partial Z^{2}}=0
$$

with the following boundary conditions:

$$
\begin{gathered}
\left.\frac{\partial T_{\mathrm{e}}^{*}}{\partial X}\right|_{X=0}=\left.\frac{\partial T_{\mathrm{e}}^{*}}{\partial X}\right|_{X=1}=0 \\
\left.\frac{\partial T_{\mathrm{e}}^{*}}{\partial Y}\right|_{Y=0}=\left.\frac{\partial T_{\mathrm{e}}^{*}}{\partial Y}\right|_{Y=1}=0 \\
\left.\frac{\partial T_{\mathrm{e}}^{*}}{\partial Z}\right|_{Z=0}=\frac{c}{H_{\mathrm{p}}} \frac{\lambda_{\mathrm{eq}, \mathrm{e}}}{\lambda_{\mathrm{s}}} T_{\mathrm{e}}^{*}=D_{\mathrm{e}} T_{\mathrm{e}}^{*} \\
\phi_{\mathrm{e}}(X, Y)=\left.\frac{\partial T_{\mathrm{e}}^{*}}{\partial Z}\right|_{Z=1}=\left\{\begin{array}{l}
1 \text { in the evaporator area } \\
0 \text { outside the evaporator area }
\end{array}\right.
\end{gathered}
$$

$T_{\mathrm{c}}^{*}=\lambda_{\mathrm{s}}\left(T_{\mathrm{c}}-T_{\mathrm{sat}}\right) / \varphi_{0} \mathrm{C}$ is the non-dimensional temperature solution of the 3D steady-state heat conduction equation:

$$
\frac{\partial^{2} T_{\mathrm{c}}^{*}}{\partial X^{2}}+\frac{1}{B^{2}} \frac{\partial^{2} T_{\mathrm{c}}^{*}}{\partial Y^{2}}+\frac{1}{C^{2}} \frac{\partial^{2} T_{\mathrm{c}}^{*}}{\partial Z^{2}}=0
$$

with the following boundary conditions:

$$
\begin{aligned}
& \left.\frac{\partial T_{\mathrm{c}}^{*}}{\partial X}\right|_{X=0}=\left.\frac{\partial T_{\mathrm{c}}^{*}}{\partial X}\right|_{X=1}=0 \\
& \left.\frac{\partial T_{\mathrm{c}}^{*}}{\partial Y}\right|_{Y=0}=\left.\frac{\partial T_{\mathrm{c}}^{*}}{\partial Y}\right|_{Y=1}=0
\end{aligned}
$$




$$
\begin{aligned}
& \left.\frac{\partial T_{\mathrm{c}}^{*}}{\partial Z}\right|_{Z=0}=\frac{c}{H_{\mathrm{p}}} \frac{\lambda_{\mathrm{eq}, \mathrm{c}}}{\lambda_{\mathrm{s}}} T_{\mathrm{c}}^{*}=D_{\mathrm{c}} T_{\mathrm{c}}^{*} \\
& \phi_{\mathrm{c}}(X, Y)=\left.\frac{\partial T_{\mathrm{c}}^{*}}{\partial Z}\right|_{Z=1}= \begin{cases}-\eta \text { in the condenser area } \\
0 \quad \text { outside the condenser area }\end{cases}
\end{aligned}
$$

$\lambda_{\text {eq, e }}$ and $\lambda_{\text {eq, c }}$ are respectively the equivalent thermal conductivities during evaporation and condensation. $\phi_{\mathrm{e}}(X, Y)$ is the non-dimensional heat flux of the heat sources and $\phi_{\mathrm{c}}(X, Y)$ is the non-dimensional heat flux of the heat sinks.

Depending on the boundary conditions, the non-dimensional temperature $T^{*}$ can be expanded in a form of an infinite Fourier series:

$$
\begin{aligned}
T^{*} & =\sum_{m=1}^{\infty}\left[A_{\mathrm{m} 0, \mathrm{e}}(Z)+A_{\mathrm{m} 0, \mathrm{c}}(Z)\right] \cos (m \pi X) \\
& +\sum_{n=1}^{\infty}\left[A_{0 \mathrm{n}, \mathrm{e}}(Z)+A_{0 \mathrm{n}, \mathrm{c}}(Z)\right] \cos (n \pi Y) \\
& +\sum_{n=1}^{\infty} \sum_{m=1}^{\infty}\left[A_{\mathrm{mn}, \mathrm{e}}(Z)+A_{\mathrm{mn}, \mathrm{c}}(Z)\right] \cos (m \pi X) \cos (n \pi Y)
\end{aligned}
$$

The non-dimensional heat flux can be written using the following expression:

$$
\begin{aligned}
\phi(X, Y) & =\sum_{m=1}^{\infty}\left[B_{\mathrm{m} 0, \mathrm{e}}+B_{\mathrm{m} 0, \mathrm{c}}\right] \cos (m \pi X) \\
& +\sum_{n=1}^{\infty}\left[B_{0 \mathrm{n}, \mathrm{e}}+B_{0 \mathrm{n}, \mathrm{c}}\right] \cos (n \pi Y) \\
& +\sum_{n=1}^{\infty} \sum_{m=1}^{\infty}\left[B_{\mathrm{mn}, \mathrm{e}}+B_{\mathrm{mn}, \mathrm{c}}\right] \cos (m \pi X) \cos (n \pi Y)
\end{aligned}
$$


The expressions of $A_{\mathrm{m} 0, \mathrm{e}}, A_{0 \mathrm{n}, \mathrm{e}}, A_{\mathrm{mn}, \mathrm{e}}, A_{\mathrm{m} 0, \mathrm{c}}, A_{0 \mathrm{n}, \mathrm{c}}, A_{\mathrm{mn}, \mathrm{c}}, B_{\mathrm{m} 0, \mathrm{e}}, B_{0 \mathrm{n}, \mathrm{e}}$, $B_{\mathrm{mn}, \mathrm{e}}, B_{\mathrm{m} 0, \mathrm{c}}, B_{0 \mathrm{n}, \mathrm{c}}$ and $B_{\mathrm{mn}, \mathrm{c}}$ are given in Appendix.

\subsection{Hydrodynamic model and analytical solution}

Based on the model by Lefèvre and Lallemand (1) and the new boundary conditions, the relative pressure field of the liquid can be expressed in a Fourier series as well:

$$
\begin{aligned}
P_{1}=\frac{\mu_{\mathrm{l}}}{K h_{\mathrm{lv}} \rho_{\mathrm{l}} H_{\mathrm{p}} \xi}[ & \sum_{m=1}^{\infty} C_{\mathrm{m} 0} \cos (m \pi X)+\sum_{n=1}^{\infty} C_{0 \mathrm{n}} \cos (n \pi Y) \\
& \left.+\sum_{n=1}^{\infty} \sum_{m=1}^{\infty} C_{\mathrm{mn}} \cos (m \pi X) \cos (n \pi Y)\right]
\end{aligned}
$$

This equation is obtained by introducing Darcy's law into the liquid mass balance assuming a 2D flow. A similar approach was adopted for the vapor to calculate the pressure field inside the FMHP. The vapor is assumed to be laminar between two parallel plates. Combining this assumption with the mass balance for the vapor yields:

$$
\begin{array}{r}
P_{\mathrm{v}}=-\frac{12}{H_{\mathrm{v}}^{3}} \frac{\mu_{\mathrm{v}}}{h_{\mathrm{lv}} \rho_{\mathrm{v}}}\left[\sum_{m=1}^{\infty} C_{\mathrm{m} 0} \cos (m \pi X)+\sum_{n=1}^{\infty} C_{0 \mathrm{n}} \cos (n \pi Y)\right. \\
\left.+\sum_{n=1}^{\infty} \sum_{m=1}^{\infty} C_{\mathrm{mn}} \cos (m \pi X) \cos (n \pi Y)\right]
\end{array}
$$

The expressions of $C_{\mathrm{m} 0}, C_{0 \mathrm{n}}$ and $C_{\mathrm{mn}}$ are given in Appendix.

The maximum heat transfer capability of the FMHP (capillary limit) is reached when the following expression is verified:

$$
\Delta P_{\text {cap }}=\Delta P_{1}+\Delta P_{\mathrm{v}}+\Delta P_{\mathrm{g}}
$$


where the subscript $\mathrm{g}$ is related to gravitational forces and $\Delta P_{\text {cap }}$ is the capillary pressure inside the FMHP.

The capillary pressure can be calculated using the following classical expression, which results from the Young-Laplace equation:

$$
\Delta P_{\text {cap }}=\sigma\left(\frac{1}{r_{1}}+\frac{1}{r_{2}}\right)
$$

in which $\sigma$ is the surface tension of the fluid.In case of rectangular microgrooves, $r_{2}=\infty$ and $r_{1}$ is the minimum meniscus curvature radius defined as:

$$
r_{1}=\frac{l_{\mathrm{g}}}{2 \cos \theta}
$$

with $l_{\mathrm{g}}$ the groove width and $\theta$ the wall/liquid contact angle.

\section{Expressions for equivalent thermal conductivity of rectangular grooves}

The FMHP analytical model will be validated with experimental data obtained by Rullière et al. (6) who studied a FMHP with rectangular grooves. Hence, expressions of the equivalent thermal conductivity for rectangular grooves have to be calculated for evaporation and condensation. The groove parameters, presented in Fig. 2 are the fin width $l_{\mathrm{f}}$ and the groove height and width, $H_{\mathrm{g}}$ and $l_{\mathrm{g}}$. 


\subsection{Expressions from the literature}

Some expressions can be found for different capillary structures in the literature. For condensation, Faghri (14) recommends to use the solution for the case of parallel heat conduction in both the fin and the liquid in the groove:

$$
\lambda_{\text {eq, }}=\frac{l_{\mathrm{g}} \lambda_{\mathrm{l}}+l_{\mathrm{f}} \lambda_{\mathrm{s}}}{l_{\mathrm{g}}+l_{\mathrm{f}}}
$$

Heat transfer by condensation occurs mainly on the fin top rather than in the grooves because the thermal resistance of the fins is much lower than the liquid thermal resistance. Thus, a liquid film overlays the fin top. Actually, the fins are generally made of a high thermal conductivity material, which is about 100 to 1000 times higher than the liquid thermal conductivity. Depending on its thickness, the condensing film thermal resistance is not negligible and can be higher than the wall resistance. In Eq. 22, this film is not taken into account, which over-estimates the equivalent conductivity in condensation.

Chi, cited in (14) has developed a correlation to calculate the groove equivalent conductivity during evaporation:

$$
\lambda_{\text {eq, e }}=\frac{\left(l_{\mathrm{f}} \lambda_{\mathrm{l}} \lambda_{\mathrm{s}} H_{\mathrm{g}}\right)+\left(l_{\mathrm{g}} \lambda_{\mathrm{l}}\right)\left(0.185 l_{\mathrm{f}} \lambda_{\mathrm{s}}+H_{\mathrm{g}} \lambda_{\mathrm{l}}\right)}{\left(l_{\mathrm{g}}+l_{\mathrm{f}}\right)\left(0.185 l_{\mathrm{f}} \lambda_{\mathrm{s}}+H_{\mathrm{g}} \lambda_{\mathrm{l}}\right)}
$$

This expression does not take into account the meniscus curvature radius. However, heat transfer by evaporation mainly occurs at the junction between the meniscus and the fin. The larger the radius of curvature, the lower is the equivalent conductivity.

As it has been shown above, literature expressions for equivalent conductivities 
of micro-grooves in the evaporator and condenser sections are not suitable. This is the reason why we built a numerical database to calculate $\lambda_{\text {eq }}$ in methanol-filled groove structures. This database includes values of $\lambda_{\text {eq }}$ for a large number of conditions (various geometries, sizes, meniscus radius...) and is presented under the form of dimensionless correlations. It was obtained from a specific $2 \mathrm{D}$ thermal model of a grooved capillary structure that was developed and validated by Lefèvre et al. (11) on one hand for evaporation (Fig. 2) and on the other hand for condensation (Fig. 3).

\subsection{Equivalent thermal conductivity during evaporation}

As regards the evaporator cross-section (Fig. 2), a constant heat flux $\varphi_{0}$ is imposed at the wall. At the fin top, the heat flux is assumed to be equal to zero, because the convection heat transfer between the wall and the vapor is negligible compared to the evaporation heat transfer. The heat transfer is equal to zero on the vertical boundaries of the control volume because of the symmetries. As the liquid thickness and the liquid velocity are small, liquid convection is neglected in the grooves and heat transfer in the liquid is modeled by $2 \mathrm{D}$ heat conduction. At the liquid-vapor interface a Fourier condition is used to model the evaporation with a heat transfer coefficient $h_{\text {int }}$ calculated from the gas kinetic theory (Carey (15)):

$$
h_{\mathrm{int}}=\frac{2 a_{\mathrm{c}}}{2-a_{\mathrm{c}}} \frac{\rho_{\mathrm{v}} h_{\mathrm{lv}}^{2}}{T_{\mathrm{sat}}}\left(2 \pi \frac{\bar{R}}{\bar{M}} T_{\mathrm{sat}}\right)^{-1 / 2}\left(1-\frac{P_{\mathrm{sat}}}{2 \rho_{\mathrm{v}} h_{\mathrm{lv}}}\right)
$$

where $a_{\mathrm{c}}$ is the accommodation coefficient, $\bar{R}$ the universal gas constant and $\bar{M}$ the molecular weight. For methanol, $a_{\mathrm{c}}$ is equal to 0.13 (16). 
The equivalent thermal conductivity is deduced from the resulting temperature field. In the range of parameters listed in Table 1, the results can be arranged under the form of a correlation that takes into account the geometry parameters $\left(H_{\mathrm{g}}, l_{\mathrm{g}}\right.$ and $\left.l_{\mathrm{f}}\right)$, the fluid properties, the evaporation heat transfer coefficient derived from the gas kinetic theory $\left(h_{\text {int }}\right)$ and also the meniscus curvature radius $R$. The resulting temperature field is independent of the heat flux so that $\varphi_{0}$ is not included in the correlation. The constant and exponents of the correlation were obtained by the least square method and the resulting expression is as follows:

$$
\lambda_{\text {eq, } \mathrm{e}}=4.5 \lambda_{\mathrm{l}}\left(\frac{H_{\mathrm{g}}}{l_{\mathrm{g}}+l_{\mathrm{f}}}\right)\left(\frac{h_{\text {int }} l_{\mathrm{g}}}{\lambda_{\mathrm{l}}}\right)^{0.14}\left(\frac{R}{l_{\mathrm{g}}}\right)^{-0.23}
$$

As shown in Fig. 4, the correlation predicts $100 \%$ of the data within a $\pm 20 \%$ error band with a mean absolute error (MAE) of $2.2 \%$ and a mean relative error (MRE) of $-0.7 \%$. The prediction is thus very good.

For the geometry tested by Rullière et al. (6), i.e. $l_{\mathrm{g}}=400 \mu \mathrm{m}, l_{\mathrm{f}}=400 \mu \mathrm{m}$ and $H_{\mathrm{g}}=380 \mu \mathrm{m}$ at $T_{\mathrm{sat}}=70^{\circ} \mathrm{C}$, the value of the equivalent thermal conductivity given by Faghri (Eq. 23) is around $6 \mathrm{~W} / \mathrm{m} . \mathrm{K}$ whereas the present correlation gives a value around $1.3 \mathrm{~W} / \mathrm{m} . \mathrm{K}$ (for $R=400 \mu \mathrm{m}$ ). The difference is thus significant, emphasizing the necessity of developing new prediction methods for determining the equivalent thermal conductivities during evaporation.

\subsection{Equivalent thermal conductivity during condensation}

In the condenser section, the condensation of the vapor occurs mainly on the top of the fin rather than in the grooves because of the high thermal 
conductivity of the FMHP wall. For the calculation of the equivalent thermal conductivity, it is necessary to accurately know the liquid film thickness $\delta$ on the top of the fin (Fig. 3). Another specific model, namely a hydrodynamic model based on the conservation equations and the Young-Laplace law is used to calculate it (Lefèvre et al. (11)). Once the liquid film thickness on the fin is known, a specific $2 \mathrm{D}$ thermal model is used to calculate the equivalent thermal conductivity during condensation. This thermal model is similar to the specific thermal model described in Section 3.2, except that the heat flux $\varphi_{0}$ is exiting the system at the wall. A constant heat flux $\varphi_{0}$ is considered outside the wall. For the calculation of the condensation heat transfer coefficient, the gas kinetic theory is also invoked, so that Eq. 24 is used to calculate the heat transfer coefficient $h_{\text {int }}$ at the liquid-vapor interface.

The correlation has been developed from a numerical database whose range of parameters is listed in Table 2. The correlation includes the geometry parameters $\left(H_{\mathrm{g}}, l_{\mathrm{g}}\right.$ and $\left.l_{\mathrm{f}}\right)$, the fluid properties depending on the saturation temperature, the heat flux $\varphi_{0}$ and the meniscus curvature radius $R$. While the temperature field was independent of the heat flux during evaporation, it strongly depends on $\varphi_{0}$ during condensation, since the heat flux controls the liquid film thickness at the top of the fins. For the sake of simplicity of use in practice, the heat flux is preferred to the liquid film thickness as a parameter of the correlation. The coefficients have been determined using the least square method. The correlation is as follows:

$$
\lambda_{\mathrm{eq}, \mathrm{c}}=16 \lambda_{\mathrm{l}}\left(\frac{H_{\mathrm{g}}}{l_{\mathrm{g}}+l_{\mathrm{f}}}\right)\left(\frac{\rho_{\mathrm{v}} h_{\mathrm{lv}}\left(\frac{g\left(\rho_{\mathrm{l}}-\rho_{\mathrm{v}}\right) \sigma}{\rho_{\mathrm{v}}^{2}}\right)^{1 / 4}}{\eta \varphi_{0}}\right)^{0.22}\left(\frac{R}{l_{\mathrm{g}}}\right)^{-0.1}\left(\frac{l_{\mathrm{f}}}{l_{\mathrm{g}}+l_{\mathrm{f}}}\right)^{0.14}
$$


As shown in Fig. 5, the correlation predicts $100 \%$ of the data within a $\pm 20 \%$ error band with a MAE of $3.9 \%$ and a MRE of $-0.7 \%$. The prediction is thus as good as for $\lambda_{\text {eq, e }}$.

For the FMHP of Rullière et al. (6) during condensation, the equivalent thermal conductivity calculated with Faghri's correlation (Eq. 22) would be around $190 \mathrm{~W} / \mathrm{m} . \mathrm{K}$ whereas the present correlation gives a value equal to $3.3 \mathrm{~W} / \mathrm{m} . \mathrm{K}$ (for $R=1600 \mu \mathrm{m}$ ). Two orders of magnitude separates both values which highlights the necessity for new and accurate methods for the calculation of $\lambda_{\text {eq }}$.

\subsection{Coupling of the hydrodynamic and thermal models}

The key to the use of the correlations is the evaluation of the curvature radius $R$ along the grooves and especially in the evaporator and condenser regions. The value of $R$ is not known a priori. In working conditions, it varies from the evaporator to the condenser because of the evaporating and condensing phenomena. However, the value of $R$ is known in non-working condition since it depends on the properties of the wall and the liquid. It is generally calculated from the value of the contact angle between the meniscus and the wall, which is a characteristic of the solid/liquid pair. In the experimental work used to validate the present model, the meniscus curvature radius was measured in non-working conditions. Its value $R_{0}$ is equal to $850 \mu \mathrm{m}$ which corresponds to a contact angle of $76.4^{\circ}$ for a groove width of $400 \mu \mathrm{m}$. It is assumed that in operating conditions $R$ remains constant at the coordinate $x_{0}$ corresponding to the section where no phase change occurs (transition between evaporation and condensation phenomena). This hypothesis allows the calculation of the meniscus curvature radii all along the grooves with the hydrodynamic model. 
The hydrodynamic model allows the calculation of the pressure field in both the liquid and vapor phases. However, the pressure fields are expressed in terms of relative pressure with respect to a reference pressure value. For the vapor, the reference pressure is the minimum pressure, which is supposed to be equal to the saturation pressure. For the liquid, the reference pressure is calculated at the coordinate $x_{0}$ with the Young-Laplace law and $R_{0}$ :

$$
P_{1}=P_{\mathrm{v}}-\frac{\sigma}{R_{0}}
$$

Once the absolute pressure fields are calculated for both the liquid and the vapor, the meniscus curvature radii all along the grooves are deduced from the Young-Laplace law. The meniscus curvature radii used for the calculation of both the evaporator and condenser equivalent conductivity are taken at the middle of the evaporator and condenser, respectively.

The thermal and hydrodynamic models are solved in an iterative process since the meniscus curvature radii in the evaporator and the condenser are not known initially. After few iterations the model converges.

\section{Results and discussion}

\subsection{Experimental setup and geometrical parameters}

In this section, a comparison between the model results and the experimental data presented in (6) is presented. The FMHP is a long grooved FMHP with rectangular channels, as shown in Fig. 6. It is grooved only on its lower face, the upper face being sealed with a glass plate, which allows the liquid/vapor 
meniscus observation in the grooves. A confocal microscope is used to locate the meniscus and to measure its curvature radius in the grooves, the FMHP being in horizontal orientation. The FMHP is made of 109 longitudinal microgrooves, machined in a copper plate of area $230 \times 90 \mathrm{~mm}^{2}$. The heat source is a thick resistor film of dimensions $190 \times 90 \mathrm{~mm}^{2}$ located on the copper wall. The heat sink is a water heat exchanger of dimensions $30 \times 90 \mathrm{~mm}^{2}$. The heat source and the heat sink are separated by a short adiabatic area of length equal to $10 \mathrm{~mm}$. Two series of seven thermistors are located symmetrically along the FMHP wall and their values are averaged in each section. The FMHP is filled with methanol.

It is interesting to emphasize, as explained in Section 2.1, that this configuration is not completely adapted to the present model due to the small distance between the heat source and the heat sink. As a matter of fact, in this configuration, one part of the heat is likely transfered only by heat conduction in the wall from the heat source to the heat sink.

The input parameters of the hydrodynamic model are the porosity $\xi$ and the permeability $K$. According to Faghri (14), the porosity is given by the following relationship:

$$
\xi=\frac{l_{\mathrm{g}}}{\left(l_{\mathrm{g}}+l_{\mathrm{f}}\right)}
$$

whereas the permeability is obtained by the following expression:

$$
K=\frac{D_{\mathrm{h}}^{2} \xi}{2\left(f R e_{\mathrm{l}, \mathrm{h}}\right)}
$$


with

$$
D_{\mathrm{h}}=\frac{4 H_{\mathrm{g}} l_{\mathrm{g}}}{\left(2 H_{\mathrm{g}}+l_{\mathrm{g}}\right)}
$$

Shah and Bhatti (17) give the following expression to calculate $f R e_{1, \mathrm{~h}}$ in rectangular grooves:

$$
\begin{gathered}
f R e_{1, \mathrm{~h}}=24\left(1-1.3553 \alpha^{*}+1.9467 \alpha^{* 2}-1.7012 \alpha^{* 3}\right. \\
\left.+0.9564 \alpha^{* 4}-0.2537 \alpha^{* 5}\right)
\end{gathered}
$$

where $\alpha^{*}=\min \left(l_{\mathrm{g}} / H_{\mathrm{g}}, H_{\mathrm{g}} / l_{\mathrm{g}}\right)$.

\subsection{Comparison with experimental results}

Figure 7 shows the comparison between the simulated and experimental temperature profiles along the micro-grooves for heat fluxes ranging from 0.5 to $0.9 \mathrm{~W} / \mathrm{cm}^{2}$ ( 85.5 to $153.9 \mathrm{~W}$ ). The saturation temperature is equal to $70^{\circ} \mathrm{C}$. The agreement is very good. For a fixed heat flux, the temperatures are constant in the evaporator zone. Then, they decrease in the adiabatic zone and finally tend toward a plateau in the condenser zone. The temperature gradients are higher in the condenser than in the evaporator, which is mainly due to a larger area at the evaporator than at the condenser.

Figure 8 depicts the evolution of the meniscus curvature radius along the micro-grooves for heat fluxes equal to $0.5,0.7$ and $0.9 \mathrm{~W} / \mathrm{cm}^{2}$. The saturation temperature is equal $70^{\circ} \mathrm{C}$. The meniscus curvature radius is of the order of $l_{\mathrm{g}}$ in the heat source zone and increases along the micro-grooves up to reach a value around $1.5 \mathrm{~mm}$ in the condenser area. The higher the heat flux, 
the smaller is the curvature radius in the evaporator zone. The comparison between the experimental and the calculated meniscus curvature radii shows a good agreement. The value of the meniscus curvature radius in the adiabatic zone $\left(R_{0}\right)$ is equal to $850 \mu \mathrm{m}$.

In addition, it has been shown experimentally that the maximum heat transfer capability of the FMHP was equal to $0.9 \mathrm{~W} / \mathrm{cm}^{2}$ for a saturation temperature of $70^{\circ} \mathrm{C}$. The value of the minimum meniscus radius, reached before the dryout of the evaporator, is equal to $240 \mu \mathrm{m}$ and the corresponding contact angle is equal to $33^{\circ}$. This contact angle value, as well as the contact angle at the coordinate $x_{0}$, are assumed to remain constant for the calculation of the maximum heat transfer capability in the next section.

As a conclusion, we can say that even if the experimental FMHP of Rullière et al. (6) is the less favorable configuration for the present model due to the small distance between the heat source and the heat sink areas, the model has been thermally and hydrodynamically well validated.

\subsection{Results of the model}

In this Section, the influence of the saturation temperature and geometry on the maximum heat flux transferred by the system is presented.

Figure 9 shows the influence of the saturation temperature on the maximum heat transfer capability. The higher the saturation temperature, the higher is the maximum heat flux. When the saturation temperature increases from 40 to $90{ }^{\circ} \mathrm{C}$, the maximum heat flux is multiplied by almost 3.5 . When the temperature increases, the liquid viscosity decreases so that the liquid pres- 
sure drops decrease. Furthermore, Fig. 9 also depicts the influence of $T_{\text {sat }}$ on the thermal resistance that is calculated as the ratio between the maximum temperature difference along the FMHP and the heat rate. The higher the saturation temperature, the higher are the vapor density and the latent heat of vaporisation. As a result, $h_{\text {int }}$ increases and the thermal resistance decreases.

The influence of the groove width on the maximum heat transfer capability is shown in Fig. 10, for a constant fin width equal to $400 \mu \mathrm{m}$. The heat flux increases with the groove width because the liquid cross section increases (and thus the liquid flow rate increases for a given pressure drop). Furthermore, the overall thermal resistance increases linearly with $l_{\mathrm{g}}$ which is due to the low thermal conductivity of the liquid compared to the wall thermal conductivity. When the groove width increases from 200 to $600 \mu \mathrm{m}$, the overall thermal resistance is multiplied by 4.5 .

\section{Conclusions}

Flat micro heat pipes are very efficient thermal management systems. Lefèvre and Lallemand (1) modeled a FMHP by coupling a 2D hydrodynamic model for both the liquid and the vapor phases inside a FMHP and a 3D thermal model of heat conduction inside the FMHP wall. This analytical model has been modified and experimentally validated in this study. The modification consists of superposing two independent solutions in order to take into account the impact of evaporation or condensation processes on the equivalent thermal conductivities of the porous medium. In fact, the Lefèvre and Lallemand (1) model was based on a single constant value of equivalent thermal conductivity which is less realistic. 
The temperature, pressure and velocity fields can be determined using Fourier solutions. The model has been experimentally validated through a comparison with the temperature and meniscus curvature radius measurements obtained by Rullière et al. (6) for a grooved FMHP. Besides, two new simple correlations for the equivalent thermal conductivities during evaporation and condensation inside rectangular micro-grooves have been proposed based on a numerical database obtained from an additional specific model by Lefèvre et al. (11). The correlations determined here for methanol and rectangular grooves may be extended in the future to a wider range of geometrical parameters, fluids and wall materials. Furthermore, the influence of the saturation temperature and geometry on the maximum heat flux transferred by the system was presented.

\section{Acknowledgements}

This work was supported by the GIP-ANR in the frame of the non thematic project "INTENSIFILM" No ANR-06-BLAN-0119-03. 


\section{Appendix}

The expressions of $B_{\mathrm{m} 0, \mathrm{e}}, B_{0 \mathrm{n}, \mathrm{e}}$ and $B_{\mathrm{mn}, \mathrm{e}}$ depend on the location of the heat sources (Fig. 11). For a rectangular evaporator $i$ of coordinates $\left[a_{\mathrm{e} 1}(i), a_{\mathrm{e} 2}(i), b_{\mathrm{e} 1}(i), b_{\mathrm{e} 2}(i)\right]$, the values of $B_{\mathrm{m} 0, \mathrm{e}}, B_{0 \mathrm{n}, \mathrm{e}}$ and $B_{\mathrm{mn}, \mathrm{e}}$ are equal to :

$$
\begin{gathered}
B_{\mathrm{m} 0, \mathrm{e}}=\frac{2}{m \pi b}\left\{\sin \left[\frac{m \pi a_{\mathrm{e} 2}(i)}{a}\right]-\sin \left[\frac{m \pi a_{\mathrm{e} 1}(i)}{a}\right]\right\}\left[b_{\mathrm{e} 2}(i)-b_{\mathrm{e} 1}(i)\right] \\
B_{\mathrm{on}, \mathrm{e}}=\frac{2}{n \pi a}\left\{\sin \left[\frac{n \pi b_{\mathrm{e} 2}(i)}{b}\right]-\sin \left[\frac{n \pi b_{\mathrm{e} 1}(i)}{b}\right]\right\}\left[a_{\mathrm{e} 2}(i)-a_{\mathrm{e} 1}(i)\right] \\
B_{\mathrm{mn}, \mathrm{e}}=\frac{4}{m n \pi^{2}}\left\{\sin \left[\frac{m \pi a_{\mathrm{e} 2}(i)}{a}\right]-\sin \left[\frac{m \pi a_{\mathrm{e} 1}(i)}{a}\right]\right\} \\
\quad\left\{\sin \left[\frac{n \pi b_{\mathrm{e} 2}(i)}{b}\right]-\sin \left[\frac{n \pi b_{\mathrm{e} 1}(i)}{b}\right]\right\}
\end{gathered}
$$

The expressions of $B_{\mathrm{m} 0, \mathrm{c}}, B_{0 \mathrm{n}, \mathrm{c}}$ and $B_{\mathrm{mn}, \mathrm{c}}$ depend on the location of the heat sinks (Fig. 11). For a rectangular condenser $i$ of coordinates $\left[a_{\mathrm{c} 1}(i), a_{\mathrm{c} 2}(i), b_{\mathrm{c} 1}(i), b_{\mathrm{c} 2}(i)\right]$, the values of $B_{\mathrm{m} 0, \mathrm{c}}, B_{0 \mathrm{n}, \mathrm{c}}$ and $B_{\mathrm{mn}, \mathrm{c}}$ are equal to :

$$
\begin{gathered}
B_{\mathrm{m} 0, \mathrm{c}}=-\eta \frac{2}{m \pi b}\left\{\sin \left[\frac{m \pi a_{\mathrm{c} 2}(i)}{a}\right]-\sin \left[\frac{m \pi a_{\mathrm{c} 1}(i)}{a}\right]\right\}\left[b_{\mathrm{c} 2}(i)-b_{\mathrm{c} 1}(i)\right] \\
B_{\mathrm{on}, \mathrm{c}}=-\eta \frac{2}{n \pi a}\left\{\sin \left[\frac{n \pi b_{\mathrm{c} 2}(i)}{b}\right]-\sin \left[\frac{n \pi b_{\mathrm{c} 1}(i)}{b}\right]\right\}\left[a_{\mathrm{c} 2}(i)-a_{\mathrm{c} 1}(i)\right] \\
B_{\mathrm{mn}, \mathrm{c}}=-\eta \frac{4}{m n \pi^{2}}\left\{\sin \left[\frac{m \pi a_{\mathrm{c} 2}(i)}{a}\right]-\sin \left[\frac{m \pi a_{\mathrm{c} 1}(i)}{a}\right]\right\} \\
\left\{\sin \left[\frac{n \pi b_{\mathrm{c} 2}(i)}{b}\right]-\sin \left[\frac{n \pi b_{\mathrm{c} 1}(i)}{b}\right]\right\}
\end{gathered}
$$

The expressions of the coefficients $A_{\mathrm{m} 0, \mathrm{e}}, A_{0 \mathrm{n}, \mathrm{e}}$ and $A_{\mathrm{mn}, \mathrm{e}}$ are: 


$$
A_{\mathrm{m} 0, \mathrm{e}}(Z)=\frac{B_{\mathrm{m} 0, \mathrm{e}}\left[\left(m \pi C+D_{\mathrm{e}}\right) \exp (m \pi C Z)+\left(m \pi C-D_{\mathrm{e}}\right) \exp (-m \pi C Z)\right]}{m \pi C\left[\left(m \pi C+D_{\mathrm{e}}\right) \exp (m \pi C)-\left(m \pi C-D_{\mathrm{e}}\right) \exp (-m \pi C)\right]}
$$

$$
\begin{gathered}
A_{0 \mathrm{n}, \mathrm{e}}(Z)=\frac{B_{0 \mathrm{n}, \mathrm{e}}\left[\left(n \pi C / B+D_{\mathrm{e}}\right) \exp (n \pi C Z / B)+\left(n \pi C / B-D_{\mathrm{e}}\right) \exp (-n \pi C Z / B)\right]}{n \pi C\left[\left(n \pi C+B D_{\mathrm{e}}\right) \exp (n \pi C / B)-\left(n \pi C-B D_{\mathrm{e}}\right) \exp (-n \pi C / B)\right]} \\
A_{\mathrm{mn}, \mathrm{e}}(Z)=\frac{B_{\mathrm{mn}, \mathrm{e}}\left[\left(G \pi C+D_{\mathrm{e}}\right) \exp (G \pi C Z)+\left(G \pi C-D_{\mathrm{e}}\right) \exp (-G \pi C Z)\right]}{G \pi C\left[\left(G \pi C+D_{\mathrm{e}}\right) \exp (G \pi C)-\left(G \pi C-D_{\mathrm{e}}\right) \exp (-G \pi C)\right]}
\end{gathered}
$$

where

$$
G=\sqrt{m^{2}+\left(\frac{n}{B}\right)^{2}}
$$

The expressions of the coefficients $A_{\mathrm{m} 0, \mathrm{c}}, A_{0 \mathrm{n}, \mathrm{c}}$ and $A_{\mathrm{mn}, \mathrm{c}}$ are:

$$
\begin{gathered}
A_{\mathrm{m} 0, \mathrm{c}}(Z)=\frac{B_{\mathrm{m} 0, \mathrm{c}}\left[\left(m \pi C+D_{\mathrm{c}}\right) \exp (m \pi C Z)+\left(m \pi C-D_{\mathrm{c}}\right) \exp (-m \pi C Z)\right]}{m \pi C\left[\left(m \pi C+D_{\mathrm{c}}\right) \exp (m \pi C)-\left(m \pi C-D_{\mathrm{c}}\right) \exp (-m \pi C)\right]} \\
A_{0 \mathrm{n}, \mathrm{c}}(Z)=\frac{B_{0 \mathrm{n}, \mathrm{c}}\left[\left(n \pi C / B+D_{\mathrm{c}}\right) \exp (n \pi C Z / B)+\left(n \pi C / B-D_{\mathrm{c}}\right) \exp (-n \pi C Z / B)\right]}{n \pi C\left[\left(n \pi C+B D_{\mathrm{c}}\right) \exp (n \pi C / B)-\left(n \pi C-B D_{\mathrm{c}}\right) \exp (-n \pi C / B)\right]} \\
A_{\mathrm{mn}, \mathrm{c}}(Z)=\frac{B_{\mathrm{mn}, \mathrm{c}}\left[\left(G \pi C+D_{\mathrm{c}}\right) \exp (G \pi C Z)+\left(G \pi C-D_{\mathrm{c}}\right) \exp (-G \pi C Z)\right]}{G \pi C\left[\left(G \pi C+D_{\mathrm{c}}\right) \exp (G \pi C)-\left(G \pi C-D_{\mathrm{c}}\right) \exp (-G \pi C)\right]}
\end{gathered}
$$

The expressions of the coefficients $C_{\mathrm{m} 0}, C_{0 \mathrm{n}}$ and $C_{\mathrm{mn}}$ are: 


$$
\begin{gathered}
C_{\mathrm{m} 0}=\varphi_{0}\left(\frac{a}{m \pi}\right)^{2}\left[D_{\mathrm{e}} A_{\mathrm{m} 0, \mathrm{e}}(0)+D_{\mathrm{c}} A_{\mathrm{m} 0, \mathrm{c}}(0)\right] \\
C_{0 \mathrm{n}}=\varphi_{0}\left(\frac{b}{n \pi}\right)^{2}\left[D_{\mathrm{e}} A_{0 \mathrm{n}, \mathrm{e}}(0)+D_{\mathrm{c}} A_{0 \mathrm{n}, \mathrm{c}}(0)\right] \\
C_{\mathrm{mn}}=\varphi_{0} \frac{1}{\left(\frac{m \pi}{a}\right)^{2}+\left(\frac{n \pi}{b}\right)^{2}}\left[D_{\mathrm{e}} A_{\mathrm{mn}, \mathrm{e}}(0)+D_{\mathrm{c}} A_{\mathrm{mn}, \mathrm{c}}(0)\right]
\end{gathered}
$$




\section{References}

[1] F. Lefèvre, M. Lallemand, Coupled thermal and hydrodynamic models of flat micro heat pipes for the cooling of multiple electronic components, Int. J. Heat Mass Transfer 49 (2006) 1375-1383.

[2] M. Lallemand, F. Lefèvre, Micro/mini heat pipes for the cooling of electronic devices, in: 13th Int. Heat Pipe Conference, Shangai, China, 2004, pp. 12-23.

[3] S. W. Kang, S. H. Tsai, H. C. Chen, Fabrication and test of radial grooved micro heat pipes, App. Therm. Engineering 22 (2002) 1559-1568.

[4] M. Ivanova, A. Lai, C. Gillot, N. Sillon, C. Shaeffer, F. Lefèvre, M. Lallemand, E. Fournier, Design, fabrication and test of silicon heat pipes with radial microcapillary grooves, in: ITHERM'2006, San Diego, USA, May 30-June 2, 2006.

[5] D. A. Benson, R. T. Mitchell, M. R. Tuck, D. W. Palmer, G. P. Peterson, Ultrahighcapacity micromachined heat spreaders, Microscale Thermophysical Eng. 2 (1998) 21-30.

[6] R. Rullière, F. Lefèvre, M. Lallemand, Prediction of the maximum heat transfer capability of two-phase heat spreaders - experimental validation, Int. J. Heat Mass Transfer 50 (2007) 1255-1262.

[7] J. P. Longtin, B. Badran, F. M. Gerner, A one dimensional model of micro heat pipe during steady-state operation, J. Heat Transfer 116 (1994) 709715.

[8] S. J. Kim, J. K. Seo, K. H. Do, Analytical and experimental investigation on the operational characteristics and the thermal optimization of a miniature heat pipe with a grooved wick structure, Int. J. Heat Mass Transfer 46 (2003) 2051-2063. 
[9] V. Sartre, M. C. Zaghdoudi, M. Lallemand, Effect of interfacial phenomena on evaporative heat transfer in micro heat pipes, Int. J. Therm. Sci. 39 (2000) 498-504.

[10] D. Khrustalev, A. Faghri, Thermal characteristics of conventional and flat miniature axially grooved heat pipes, J. Heat Transfer 117 (1995) $1048-1054$.

[11] F. Lefèvre, R. Rullière, G. Pandraud, M. Lallemand, Prediction of the temperature field in two-phase heat spreaders with microgrooves - experimental validation, Int. J. Heat Mass Transfer, In Press.

[12] X. Y. Huang, C. Y. Liu, The pressure and velocity fields in the wick structure of a localized heated flat plate heat pipe, Int. J. Heat Mass Transfer 39 (1996) 1325-1330.

[13] W. Qin, C. Y. Liu, Liquid flow in the anisotropic wick structure of a flat plate heat pipe under block-heating condition, Appl. Therm. Eng. 17 (1997) 339-349.

[14] A. Faghri, Heat pipe science and technology, Taylor and Francis, 1994.

[15] V. P. Carey, Liquid - vapor phase-change phenomena: An introduction to the thermophysics of vaporization and condensation processes in heat transfer equipment, Taylor and Francis, 1992.

[16] M. Maerefat, S. Fujikawa, T. Akamatsu, T. Goto, T. Mizutani, An experimental study of non-equilibrium vapour condensation in a shock-tube, Exp. in Fluids 7 (1989) 513-520.

[17] R. K. Shah, M. S. Bhatti, Laminar convective heat transfer in ducts, Handbook of single-phase convective heat transfer, New York, 1987. 


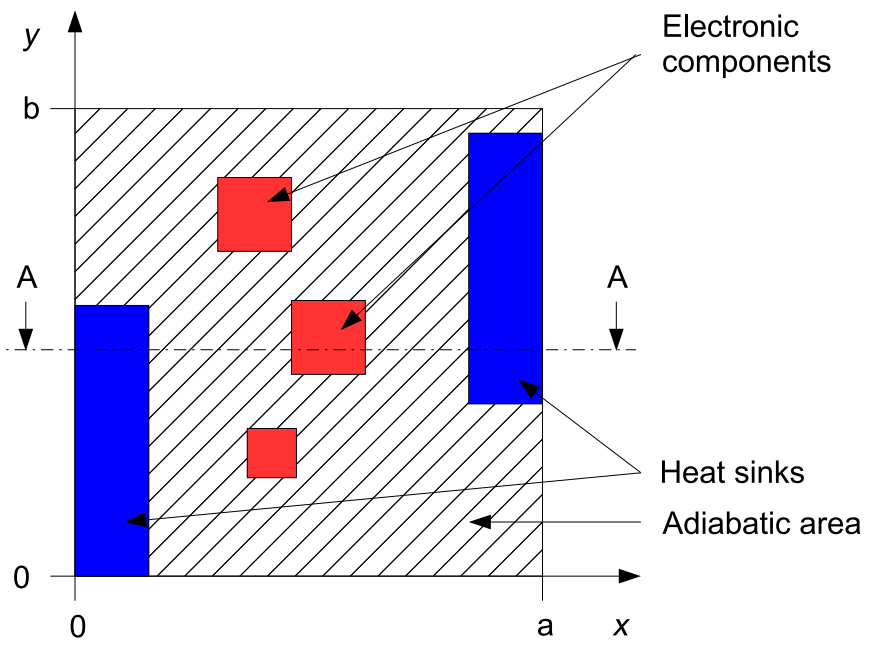

A-A

Adiabatic area

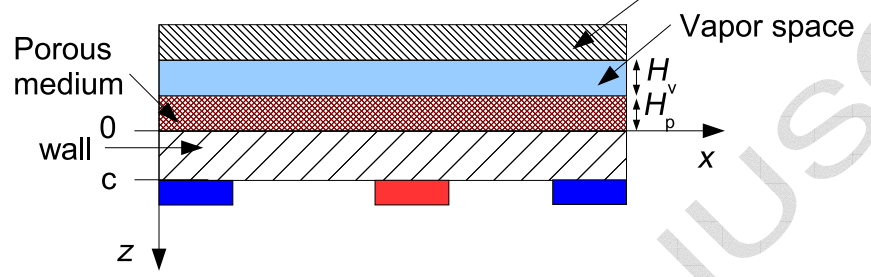

Figure 1. Schematic of a FMHP with heat sources and heat sinks. 


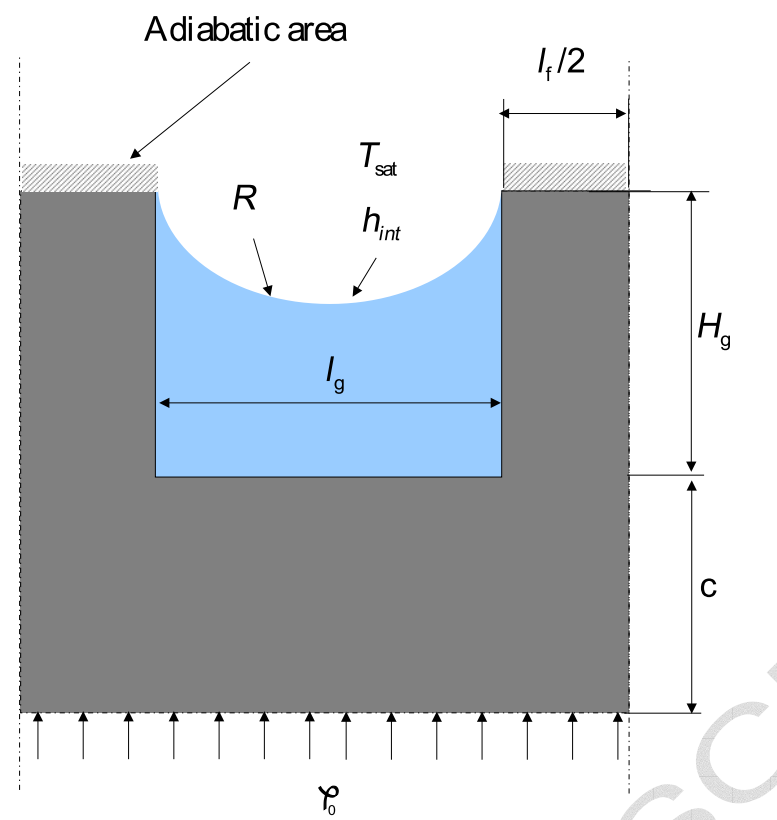

Figure 2. Schematic of the micro-groove during evaporation. 


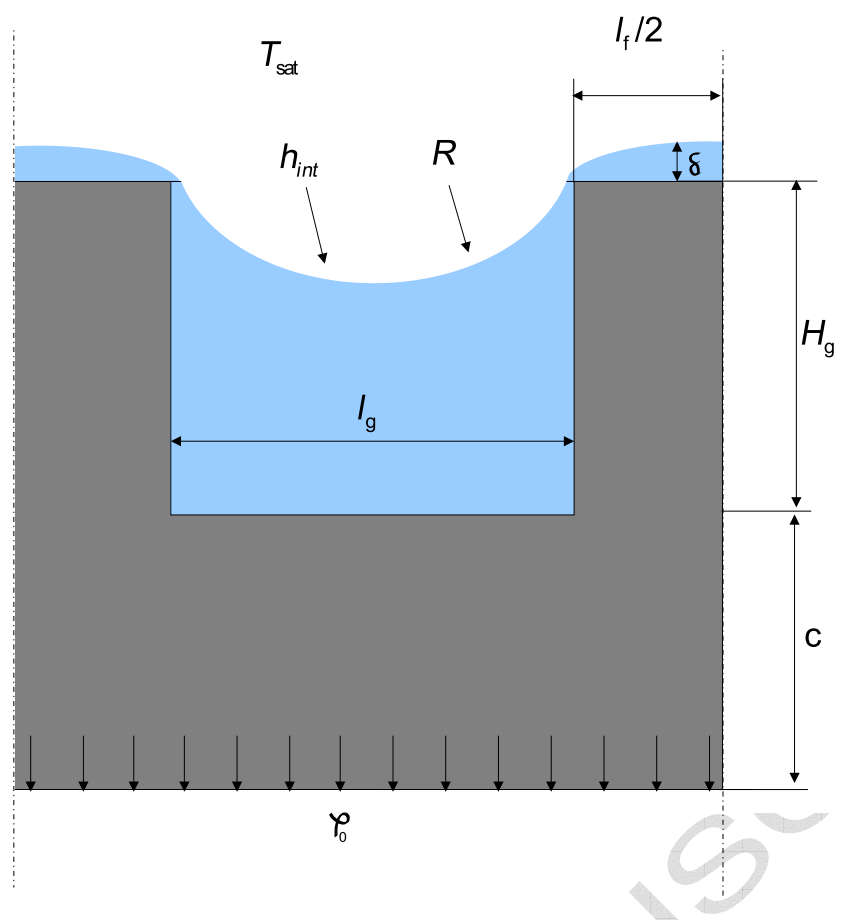

Figure 3. Schematic of the micro-groove during condensation. 


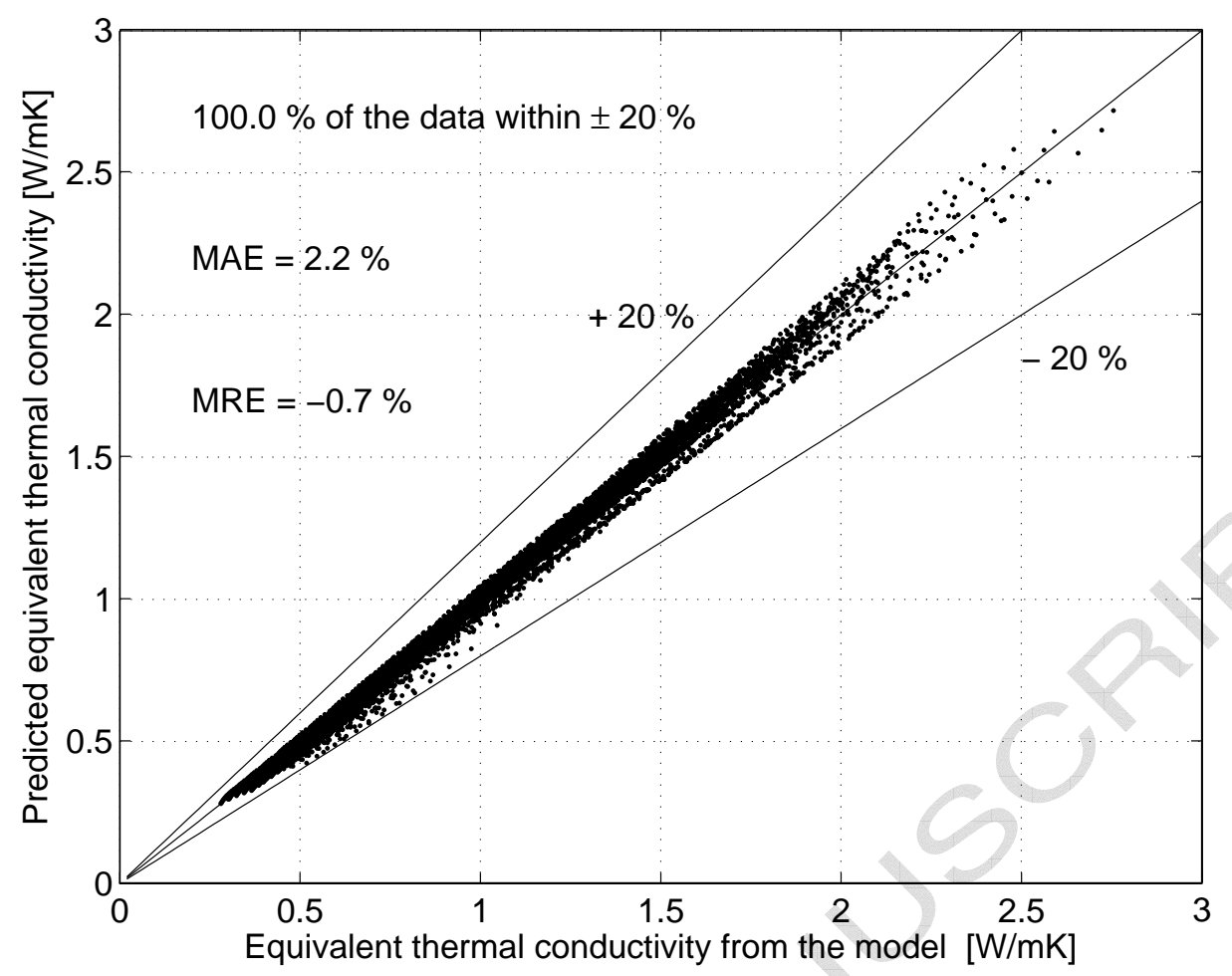

Figure 4. Comparison between the equivalent thermal conductivity from the model and the predicted thermal conductivity during evaporation. 


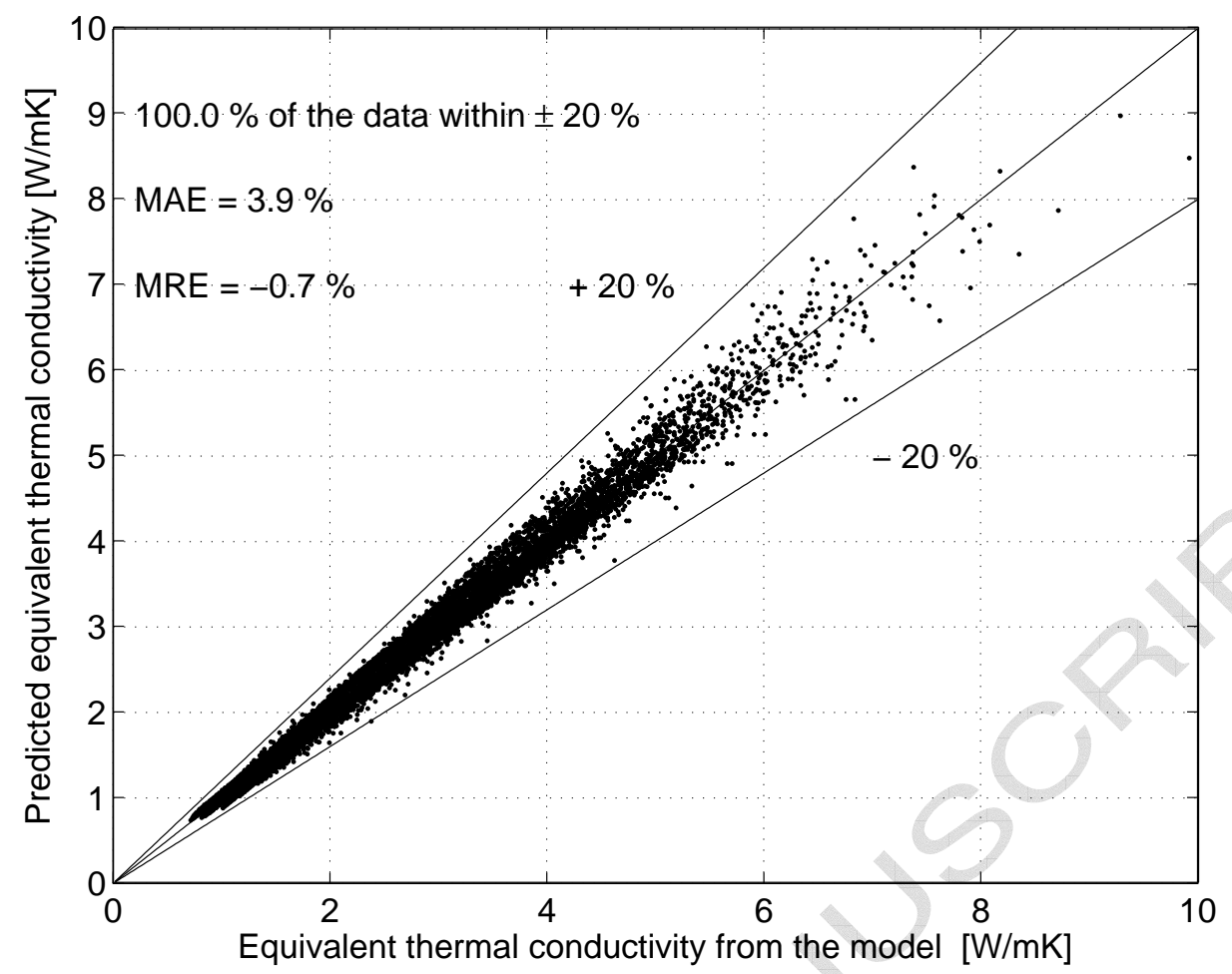

Figure 5. Comparison between the equivalent thermal conductivity from the model and the predicted thermal conductivity during condensation. 


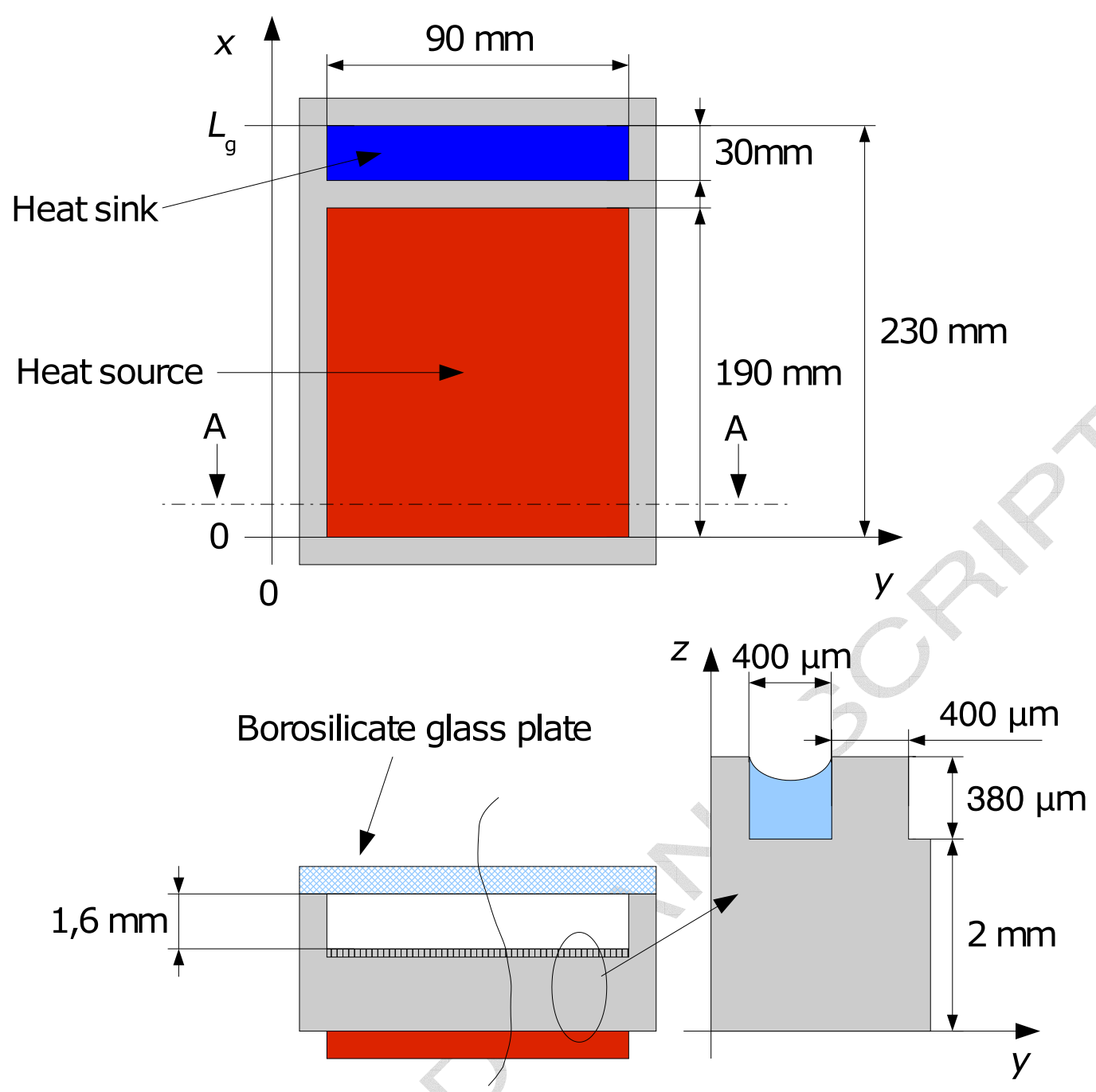

A-A

Figure 6. Schematic of the copper FMHP by Rullière et al. (6). 


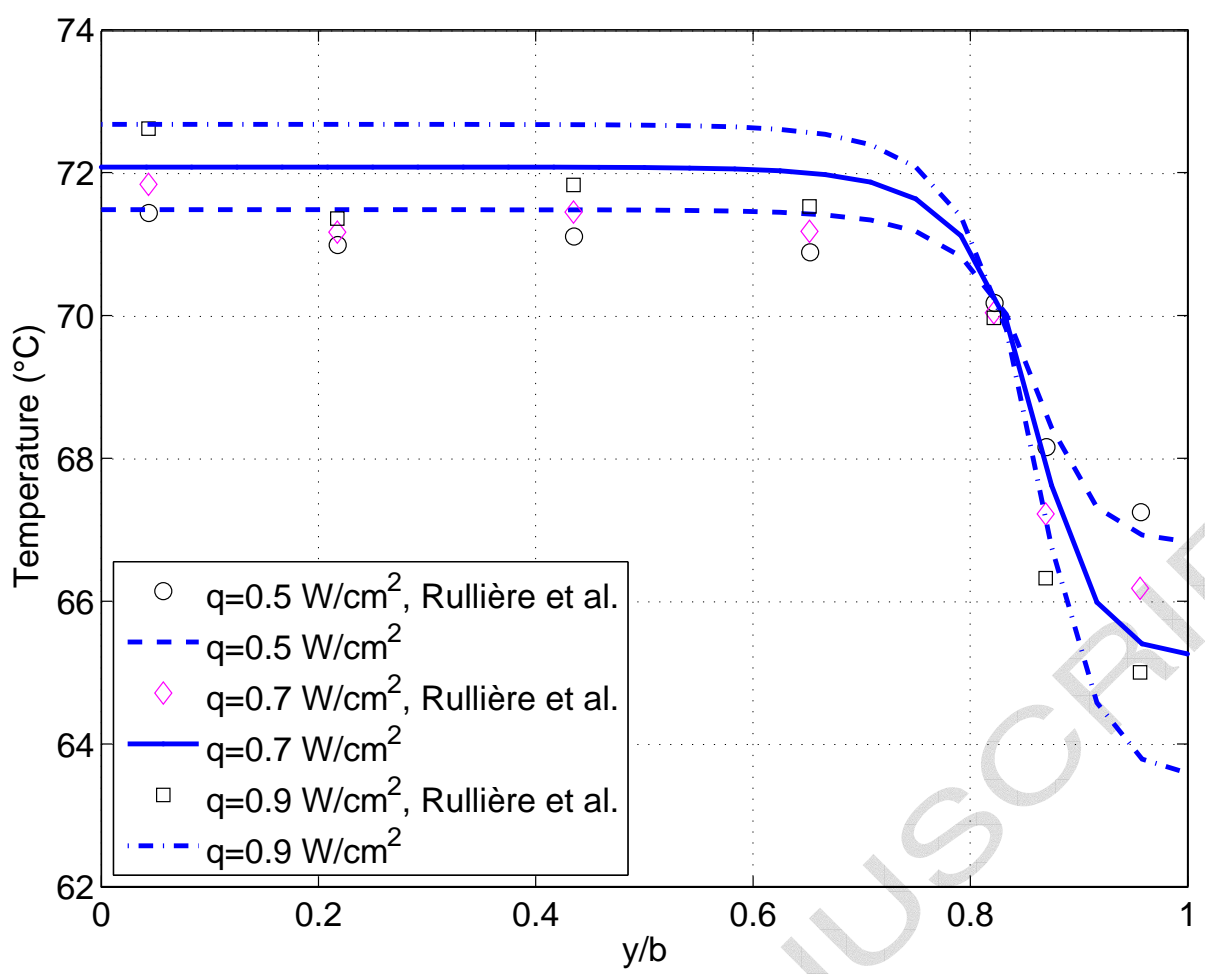

Figure 7. Comparison between the present model and the experimental results by Rullière et al. (6). 


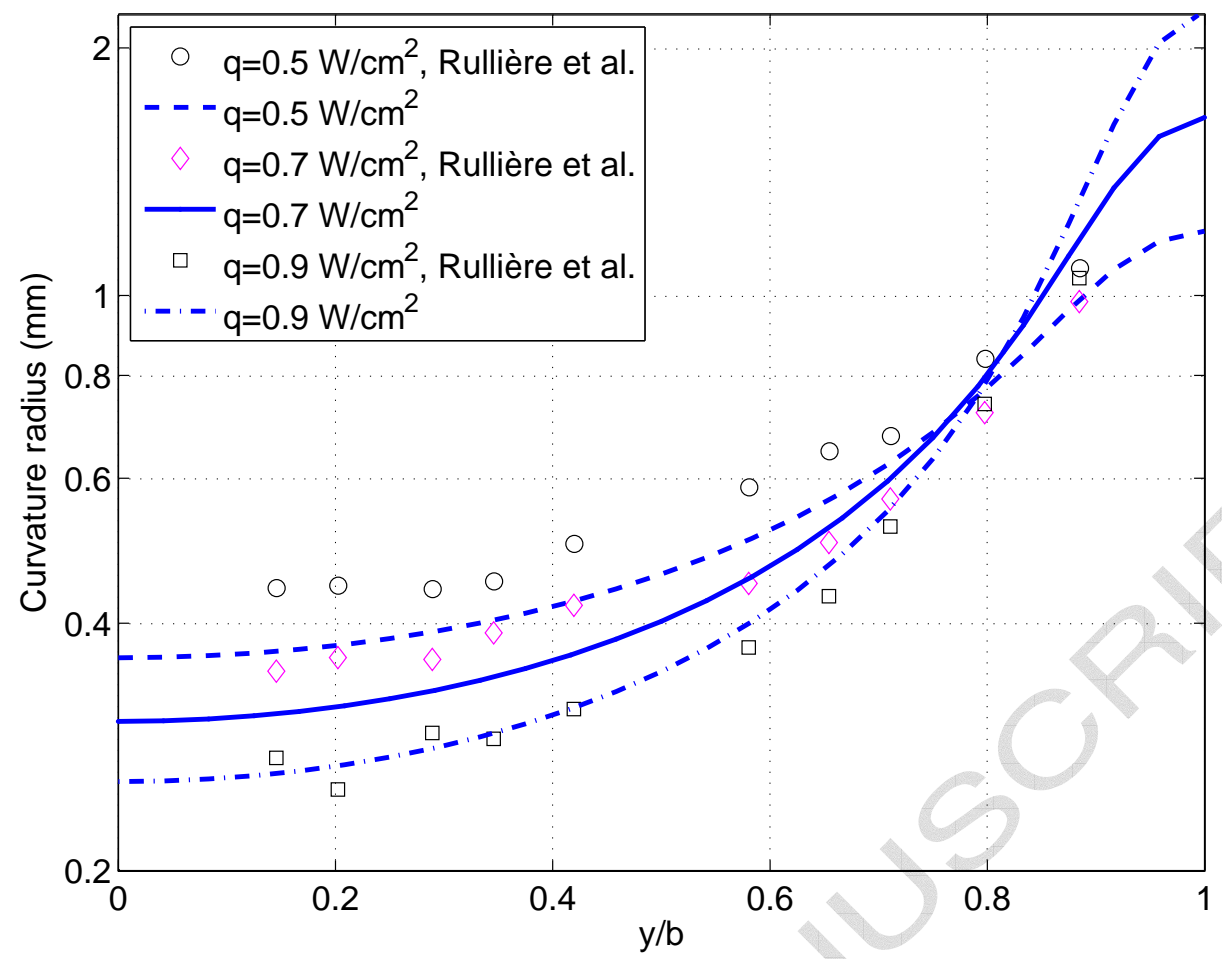

Figure 8. Comparison between the present model and the experimental results by Rullière et al. (6). 


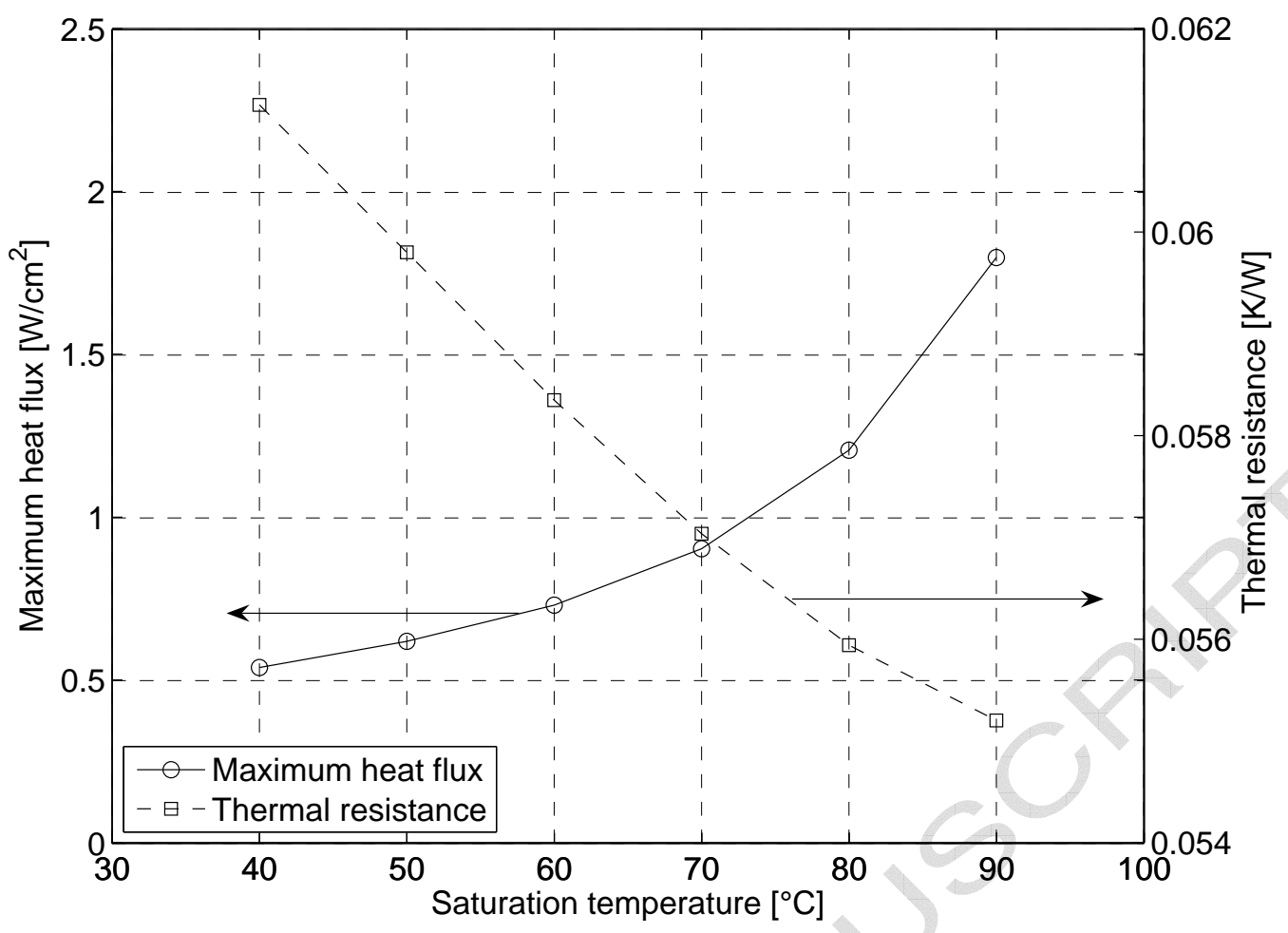

Figure 9. Effect of the saturation temperature on the maximum heat transfer capability and the overall thermal resistance. 


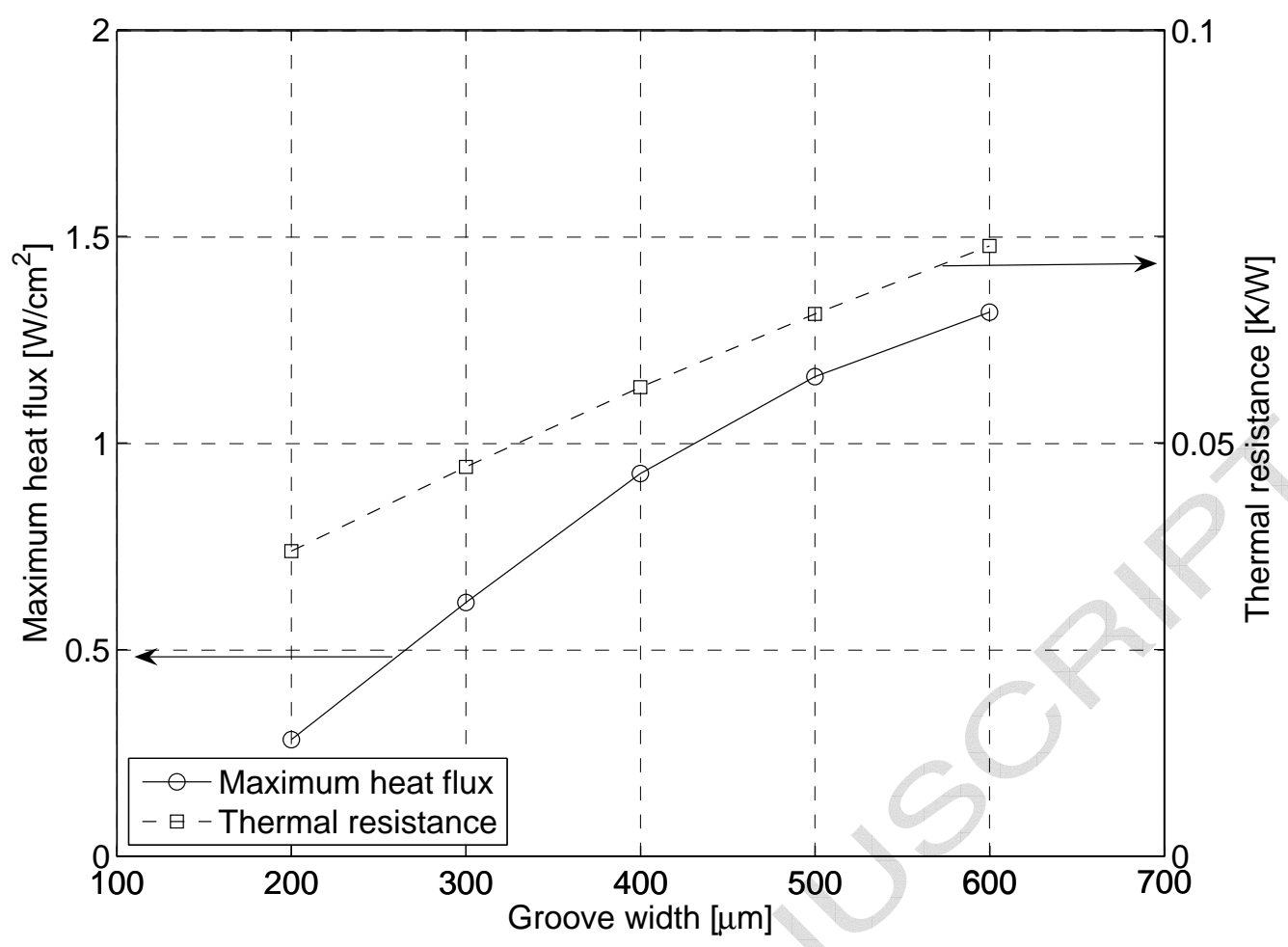

Figure 10. Effect of the groove width on the maximum heat transfer capability and the overall thermal resistance for $l_{\mathrm{f}}=400 \mu \mathrm{m}$ 


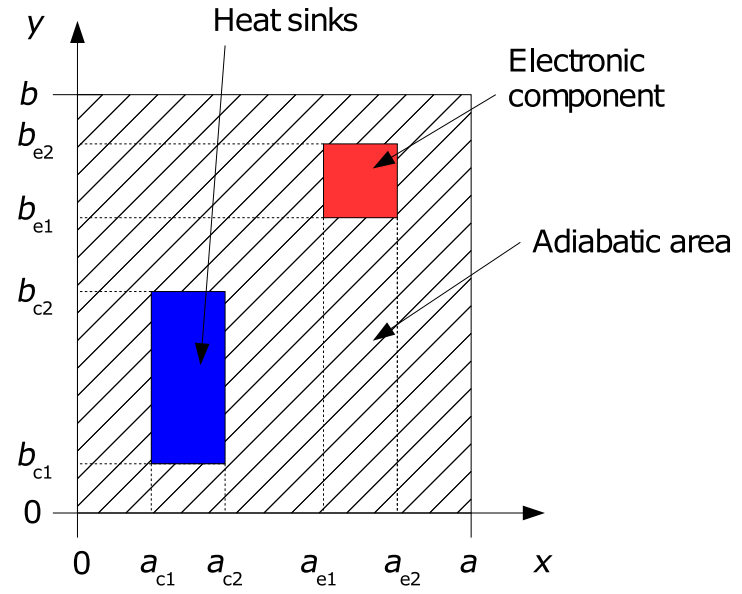

Figure 11. Coordinates of heat sources and heat sinks 


\section{List of Captions}

Figure 1: Schematic of a FMHP with heat sources and heat sinks.

Figure 2: Schematic of the micro-groove during evaporation.

Figure 3: Schematic of the micro-groove during condensation.

Figure 4: Comparison between the equivalent thermal conductivity from the model and the predicted thermal conductivity during evaporation.

Figure 5: Comparison between the equivalent thermal conductivity from the model and the predicted thermal conductivity during condensation.

Figure 6: Schematic of the copper FMHP by Rullière et al. (6).

Figure 7: Comparison between the present model and the experimental results by Rullière et al. (6).

Figure 8: Comparison between the present model and the experimental results by Rullière et al. (6).

Figure 9: Effect of the saturation temperature on the maximum heat transfer capability and the overall thermal resistance.

Figure 10: Effect of the groove width on the maximum heat transfer capability and the overall thermal resistance for $l_{\mathrm{f}}=400 \mu \mathrm{m}$.

Figure 11: Coordinates of heat sources and heat sinks. 
Table 1

Numerical database for calculating the equivalent thermal conductivity during evaporation (15000 data points).

\begin{tabular}{|c|c|c|}
\hline Parameters & Range & Units \\
\hline Fluid & Methanol & - \\
$H_{\mathrm{g}}$ & $200-600$ & $\mu \mathrm{m}$ \\
$l_{\mathrm{g}}$ & $200-600$ & $\mu \mathrm{m}$ \\
$l_{\mathrm{f}}$ & $200-600$ & $\mu \mathrm{m}$ \\
$T_{\text {sat }}$ & $40-90$ & ${ }^{\circ} \mathrm{C}$ \\
$R$ & $>\frac{7}{10} l_{\mathrm{g}}$ & $\mu \mathrm{m}$ \\
\hline
\end{tabular}


Table 2

Numerical database for calculating the equivalent thermal conductivity during (9720 data points).

\begin{tabular}{|c|c|c|}
\hline Parameters & Range & Units \\
\hline Fluid & Methanol & - \\
$H_{\mathrm{g}}$ & $200-600$ & $\mu \mathrm{m}$ \\
$l_{\mathrm{g}}$ & $200-600$ & $\mu \mathrm{m}$ \\
$l_{\mathrm{f}}$ & $200-600$ & $\mu \mathrm{m}$ \\
$T_{\mathrm{sat}}$ & $40-90$ & ${ }^{\circ} \mathrm{C}$ \\
$R$ & $l_{\mathrm{g}}-6 l_{\mathrm{g}}$ & $\mu \mathrm{m}$ \\
$\varphi_{0}$ & $0.34 \times 10^{4}-3.2 \times 10^{4}$ & $\mathrm{~W} / \mathrm{m}^{2}$ \\
\hline
\end{tabular}

